BMJ Open

Diabetes

Research

\& Care

\section{Effects of krill oil on endothelial function and other cardiovascular risk factors in participants with type 2 diabetes, a randomized controlled trial}

\author{
Jessika M Lobraico, Lauren C DiLello, Amber D Butler, Marie Elena Cordisco, \\ Joann R Petrini, Ramin Ahmadi
}

To cite: Lobraico JM, DiLello LC, Butler AD, et al. Effects of krill oil on endothelial function and other cardiovascular risk factors in participants with type 2 diabetes, a randomized controlled trial. BMJ Open Diabetes Research and Care 2015;3:e000107. doi:10.1136/bmjdrc-2015000107

Received 3 April 2015 Revised 27 August 2015 Accepted 3 September 2015

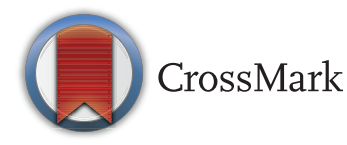

Department of Research, Western Connecticut Health Network, Danbury Hospital, Danbury, Connecticut, USA

Correspondence to Jessika M Lobraico; Jessika.Lobraico@wchn.org

\section{ABSTRACT}

Objective: The purpose of this trial was to evaluate the effect of krill oil supplementation, a source of $\omega-3$ fatty acids, on cardiovascular disease risk factors and blood glucose control among participants with type 2 diabetes.

Research design and methods: A randomized, double-blind controlled cross-over trial was employed. Outcomes assessed were: endothelial function, blood lipids, glucose, glycated hemoglobin, serum antioxidant level, $C$ peptide, and calculated Homeostatic Model Assessment of Insulin Resistance (HOMA-IR) scores. Participants were randomized to either krill oil or olive oil supplementation for 4 weeks, underwent a 2-week washout period, and then crossed to the other supplementation for 4 weeks. All participants were then offered an additional 17 weeks of krill supplementation. Testing occurred at 3 time points: baseline, after first supplementation, and after second supplementation. Testing also occurred after an optional 17 weeks of krill oil supplementation. Difference scores were calculated for each participant in both sequences (ie, differences in outcome measures in the first and second period of the sequence). The mean and SD of the scores in the 2 sequence groups were used to test for differences between treatment effects at a significance level of $p<0.05$. Results: A total of 47 participants were included in the initial cross-over study. Participants who received krill oil for 4 weeks had an improvement in their endothelial function and a reduction in blood $C$ peptide levels and HOMA scores as compared with the olive oil. A total of 34 participants completed the additional 17-week supplementation period. When compared with their respective baseline measures, these participants had a statistically significant improvement in endothelial function and blood high-density lipoprotein (HDL).

Conclusions: Krill oil may lead to moderate improvement of cardiovascular risks, specifically endothelial dysfunction and HDL in patients with type 2 diabetes.

Trial registration number: Registered with ClinicalTrials.gov: NCT02091193.

\section{INTRODUCTION}

The American Heart Association (AHA) recommends a daily intake of at least $1000 \mathrm{mg}$ of $\omega-3$ fatty acids to minimize risk
- Krill oil supplementation helps to reduce cardiovascular risk factors in patients with type 2 diabetes.

- Four weeks of krill oil supplementation improved endothelial function, reduced $C$ peptide levels, and improved insulin resistance as calculated by HOMA in patients with type 2 diabetes.

- Serum high-density lipoprotein significantly increased to more optimal levels following 17 weeks of krill oil supplementation.

- This study was designed as a randomized, double-blind trial with a cross-over design.

- The daily dose of krill oil used in this trial was $1000 \mathrm{mg}$. Are the results of this study dose dependent or could an increase or decrease in the dosage offer the same, better, or worse results?

- What mechanism is responsible for the improvement and maintenance of endothelial function after both short-term and long-term krill oil supplementation?

- How would a larger study with a longer term supplementation period (greater than 4 weeks) with krill oil compared with olive oil further impact cardiovascular risk factors in patients with type 2 diabetes?

factors associated with cardiovascular disease (CVD), even for patients at high risk of developing CVD. ${ }^{1}$ The published recommendations predated the commercial availability of krill oil, a supplement similar in composition to fish oil. Krill oil is produced from small oceanic crustaceans at the base of the food chain (krill) that primarily ingest algae rich in $\omega-3$ fatty acids. The oils produced by krill are thought to be a less polluted and more pure source of $\omega-3$ fatty acids, notably eicosapentaenoic acid (EPA) and docosahexaenoic acid (DHA), compared with other fish oils. ${ }^{2}$ EPA and DHA are the primary fatty acids that contribute toward improved cardiovascular health. ${ }^{3}$

\section{Key messages}


While both krill and fish oils possess $\omega-3$ fatty acids, the fatty acids in fish oil are attached to either triglycerides or ethyl esters which reportedly produce a high abundance of $\omega-3$ fatty acids in whole blood. ${ }^{4}$ However, it has been suggested that krill oil has better bioavailability because the $\omega-3$ fatty acids, specifically EPA and DHA, are attached to phospholipids and multiple reports have shown superior absorption in the intestines and further increased phospholipid-bound $\omega-3$ fatty acid plasma and red blood cell levels. ${ }^{4-6}$ Krill oil also contains astaxanthin, an antioxidant, which may reduce the presence of free radicals, free oxygen, and inflammation in humans. ${ }^{78}$ Oxidative stress and inflammation are known contributors to atherosclerosis, endothelial dysfunction, and overall CVD risk. ${ }^{8}$ Therefore, despite the great abundance of $\omega-3$ fatty acids available in fish oil, the delivery of fatty acids and presence of astaxanthin in krill oil may provide superior health benefits and meets the AHA recommendations of $1000 \mathrm{mg}$ of $\omega-3$ fatty acid consumption.

Patients with type 2 diabetes have a high incidence of CVD and often have traditional cardiovascular risk factors like hyperlipidemia, elevated blood glucose, insulin resistance, and hypertension. ${ }^{3}{ }^{9}$ Risk factors including endothelial dysfunction, oxidative stress, and inflammation have also been reported. ${ }^{3} 9$ Identifying opportunities for prevention of CVD progression in patients with type 2 diabetes is critically important and may be underappreciated. The purpose of this study was to evaluate the effect of krill oil supplementation on CVD risk and blood glucose control among patients with type 2 diabetes.

\section{RESEARCH DESIGN AND METHODS}

\section{Trial design}

In a randomized, double-blind trial using a cross-over design, the effect of krill oil (Prograde Inc, Lutz, Florida, USA) as compared with olive oil was evaluated in participants with type 2 diabetes. A cross-over design involves two treatments which are consecutively administered in each patient recruited for the study. ${ }^{10}$ In our study, all participants received baseline testing for key outcome measures. They were randomized to either two pure krill oil capsules daily containing $1000 \mathrm{mg}$ of $\omega-3$ fatty acids for 4 weeks or two olive oil capsules for 4 weeks, followed by a 2-week washout period, and then the groups were crossed for 4 weeks. Study visits occurred at baseline $\left(\mathrm{T}_{0}\right)$, after completion of the first supplementation period $\left(\mathrm{T}_{1}\right)$, and after completion of the second supplementation period $\left(\mathrm{T}_{2}\right.$; figure 1$)$ at the Western Connecticut Health Network Biomedical Research Institute, Danbury, Connecticut, USA.

Following the cross-over trial, participants were invited to participate in an additional 17-week krill oil supplementation period and return for assessment after completion of the long-term supplementation period.

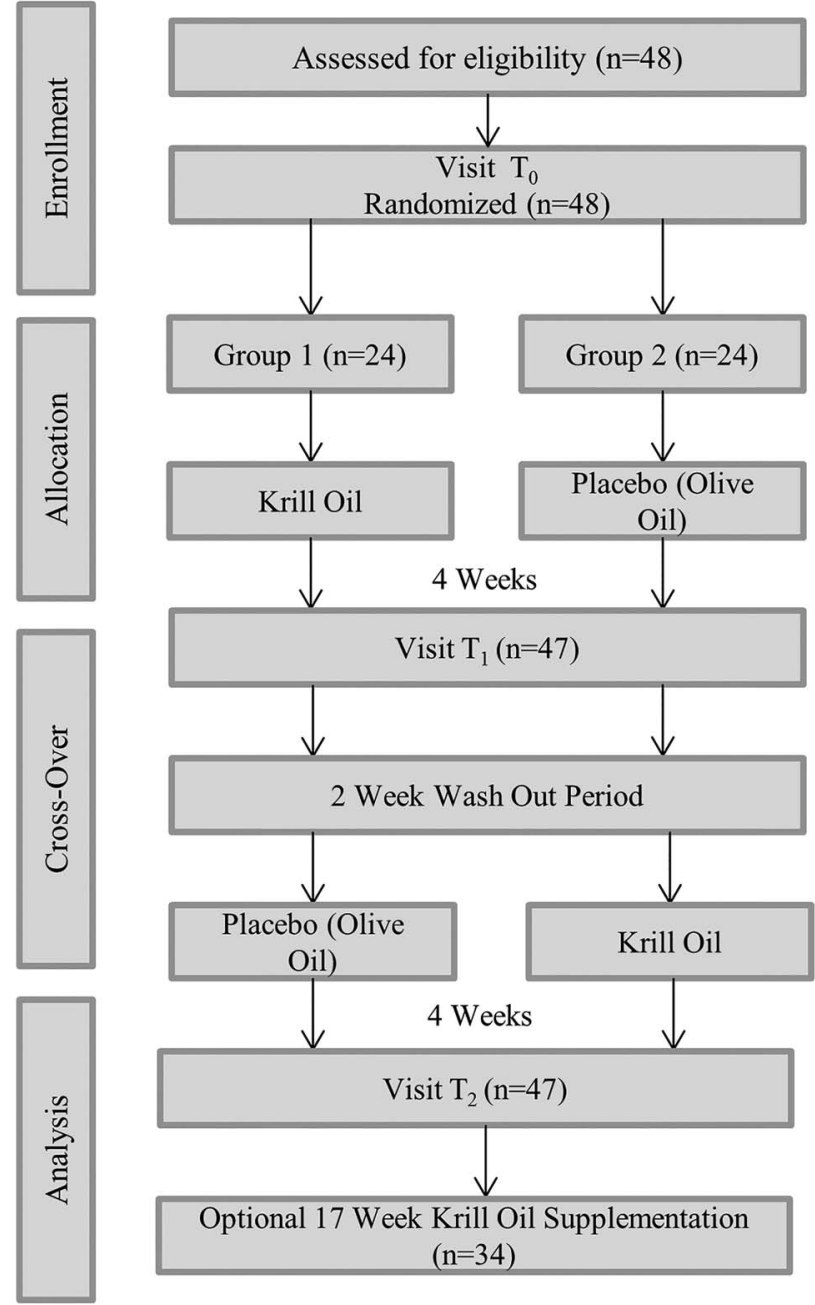

Figure 1 Cross-over study flow.

All participants received a phone call at the midpoint of each round of supplementation to evaluate compliance and adverse effects. Research staff requested that participants bring their supplement bottles to their study visits to further assess subject compliance, and any changes in medication throughout the course of the study were documented.

This study was approved by the Biomedical Research Alliance of New York Institutional Review Board. Written informed consent was obtained from all participants prior to the start of the trial after the experimental protocol had been explained to them in detail. This trial was registered at ClinicalTrials.gov (NCT02091193).

\section{Trial population}

Participants were enrolled in the trial between June 2012 and December 2013. Recruitment methods included medical provider referrals, newspaper advertisements, and flyers distributed throughout the greater Danbury, Connecticut area. Inclusion criteria included adults with a diagnosis of type 2 diabetes and on stable oral glucose-lowering agents and/or insulin that could be confirmed by a healthcare provider. Patients were 
excluded from the study if they were less than 18 years of age, were pregnant or lactating, had a blood coagulation disorder or used oral anticoagulants other than aspirin, had a seafood allergy, or consumed fish oil or krill oil supplements within 2 weeks of the start of the study. The trial protocol was reviewed with all eligible participants and written consent was obtained from each participant. Each participant's baseline medical and social history, including duration of diagnosis with type 2 diabetes and other cardiovascular risk factors, were documented at the time of enrolment.

\section{Trial visits and laboratory procedures}

The endothelial function of participants was tested at each visit using an Food and Drug Administration (FDA)-approved device, the EndoPAT2000 (Itamar Medical Inc, Franklin, Massachusetts, USA). The EndoPAT2000 is a non-invasive method to assess peripheral arterial tonometry (PAT) in the fingers. ${ }^{11}$ Endothelial function testing was conducted in a quiet, temperature-controlled room following the manufacturer's recommendations. Each participant was placed in a supine position and the PAT sensors were placed on the same finger of the left and right hands. A sphygmomanometer cuff was placed on the participant's nondominant arm and the dominant arm acted as the control. Measurement included a 5 min baseline or basal recording of PAT, 5 min of brachial artery occlusion in the non-dominant arm by inflating the blood pressure cuff, and $5 \mathrm{~min}$ of a reactive hyperemia measurement for a total of $15 \mathrm{~min}$ of testing. Brachial artery occlusion was achieved by inflating the sphygmomanometer cuff to at least $60 \mathrm{~mm} \mathrm{Hg}$ over the patient's systolic blood pressure (with a minimum of $200 \mathrm{~mm} \mathrm{Hg}$ and a maximum of $260 \mathrm{~mm} \mathrm{Hg}$ ). The Reactive Hyperemia Index (RHI) was automatically analyzed and calculated using EndoPAT2000 software, V.3.4.4. An RHI above 1.67 represents moderate risk of endothelial dysfunction and above 2.0 indicates low risk of endothelial dysfunction. Patients with an RHI below 1.67 are high risk for endothelial dysfunction.

Fasting blood ( $8 \mathrm{~h}$ minimum) specimens were collected from participants using venipuncture at the baseline visit $\left(\mathrm{T}_{0}\right)$, at the end point of each treatment $\left(\mathrm{T}_{1}\right.$ and $T_{2}$ ) and following the 17-week supplementation period if they chose to participate. The specimens were used to determine the glycated hemoglobin (HbAlc), glucose, total cholesterol (TC), low-density lipoprotein (LDL), high-density lipoprotein (HDL), and non-HDL cholesterol. Serum for lipid profiles, glucose, total antioxidant capacity, and C peptide measurements were collected in BD Vacutainer Serum Separator Tubes (SST). Serum was then stored in microcentrifuge tubes at $-80^{\circ}$ $\mathrm{C}$ until it was thawed for analysis. HbAlc analysis was performed with whole blood using a self-contained bench-top immunoassay (Seimens DCA Vantage, Siemens Medical Solutions USA, Malvern, Pennsylvania, USA). Lipid profiles and glucose were measured using
$50 \mu \mathrm{L}$ of serum loaded onto a room temperature Cholestech Lipid Profile and Glucose cassette (Alere San Diego, Inc, San Diego, California, USA). The cassette was then loaded onto the Cholestech LDX analyzer (Alere San Diego, Inc) following product protocols.

Total antioxidant capacity and $\mathrm{C}$ peptide measurements were performed at the Western Connecticut Health Network Biomedical Research Institute using commercially available ELISA kits and their specified protocols (Abcam, Cambridge, Massachusetts, USA, and Mercodia, Inc, Winston Salem, North Carolina, USA, respectively). Homeostatic Model Assessments of Insulin Resistance (HOMA2-IR) were calculated using the HOMA2-IR (V.2.2.3, University of Oxford) calculator using measured glucose and $\mathrm{C}$ peptide values. The University of Oxford's HOMA2-IR calculator allows for C peptide to be used in calculations instead of insulin because generally, $\mathrm{C}$ peptide is produced in equal amounts to insulin. C peptide is also more stable with a longer half-life than insulin, ensuring accurate measurement following a freeze-thaw cycle. ${ }^{12} 13$

\section{Power calculation and randomization}

The cross-over design used in this study maximizes sample size as each participant serves as his/her own control. An estimation of the difference in endothelial function between the participants receiving krill oil and olive oil was used to calculate a sample size that would detect a reduction in cardiovascular risk factor measures with an $\alpha$ of 0.05 and a power of $80 \%$. This required a sample size of 50 participants. Participants were block randomized by research coordinators to two equal groups using a computer-generated list prior to the start of enrollment. ${ }^{14}$ The allocation between the two groups determined whether the subject first received krill oil capsule supplements or olive oil capsule supplements. Both supplements were blinded to the trial coordinators and the participants until the completion of the trial. Participants were assigned their group on sequential enrollment and the randomized group placement.

\section{Calculations and statistical analysis}

The efficacy of olive oil and krill oil was assessed on the basis of within-participant differences between the two treatments with regard to the outcome variables. The described procedure assumes normality of the distributions to be analyzed.

To separate treatment effects from period effects, treatment effects were computed separately in the two sequence groups. In order for the procedure to be valid, we had to check the assumption of negligible carry-over effects by conducting a pretest of the data conceived by Wellek and Blettner. ${ }^{10}$ For this test, the sum of the values measured in both periods were calculated for each participant and compared across the two sequence groups by means of a test of independent samples. ${ }^{10}$ Once we confirmed that there was negligible carry-over effect, the difference between treatment effects was assessed by 
means of a standard t test for independent samples using the intraindividual differences between values measured in both periods (difference scores).

Outcomes following the optional 17-week supplementation period were compared with the respective baseline measures and analyzed using the t test. Statistical significance for this study was defined at $\mathrm{p}<0.05$. Analyses were conducted manually and using SAS V.9.3 software.

\section{RESULTS}

\section{Baseline characteristics}

A total of 48 participants were recruited to the trial and 1 patient was lost to follow-up. The mean age of participants was 64.8 years (range $47-84$ years) and $66 \%$ were male. Twenty-two participants $(46.8 \%)$ received insulin therapy throughout the study, and 15 of those 22 participants also received oral glucose-lowering agents. The remaining 25 participants only received oral glucoselowering agents. There were no reported changes in medications or doses throughout the course of this study. The demographics and baseline characteristics of participants are shown in table 1.

The results of the pretest to check for the assumption of negligible carry-over effects showed no evidence of relevant carry-over effects. Data for both krill and olive oil supplementation periods were pooled for statistical analysis of the differences between treatment effects.

\section{Effect of supplementation on end point measures}

The primary end point, endothelial function, as measured by the EndoPAT RHI for krill oil was statistically significantly higher than that of the olive oil $(p=0.025$, table 2). In addition, there was a statistically significant reduction in $\mathrm{C}$ peptide among participants when receiving krill oil compared with olive oil $(\mathrm{p}=0.029)$. However, on further analysis, there was not a reduction of $\mathrm{C}$ peptide among participants who received insulin therapy $(\mathrm{p}=0.136)$, but the reduction of $\mathrm{C}$ peptide in those that received only oral glucose-lowering agents was still observed $(\mathrm{p}=0.034)$. There was an observed reduction of insulin resistance based on the HOMA-IR score of participants after receiving krill oil compared with olive oil $(p=0.019)$. While there was a decrease in TC during the cross-over portion of the trial that approached significance, LDL, HDL, glucose, HbAlc, and the antioxidant capacity of the participants' serum did not reach statistical significance.

\section{Comorbidities and other factors}

Of the 47 participants, $72 \%$ also had a diagnosis of hypertension, $74 \%$ had a diagnosis of dyslipidemia, and $81 \%$ were taking a prescribed statin for lipid lowering. Further, $62 \%$ of participants had a diagnosis of type 2 diabetes, hypertension, and dyslipidemia. There were no significant changes in trial measures after supplementation when participants were analyzed based on the number of diagnosed comorbidities.
Table 1 Demographics and baseline characteristics of participants $(n=47)$

\begin{tabular}{ll}
\hline Parameter & Mean \pm SD \\
\hline Age (years) & $64.8 \pm 8.8$ \\
Body weight $(\mathrm{kg})$ & $93.1 \pm 19.1$ \\
Height $(\mathrm{m})$ & $1.7 \pm 0.1$ \\
BMI $\left(\mathrm{kg} / \mathrm{m}^{2}\right)$ & $30.5 \pm 6.9$ \\
Total cholesterol (mg/dL) & $153.7 \pm 32.6$ \\
HDL $(\mathrm{mg} / \mathrm{dL})$ & $44.6 \pm 13.7$ \\
LDL (mg/dL) & $78.7 \pm 28.4$ \\
TG (mg/dL) & $155.6 \pm 95.3$ \\
Glucose $(\mathrm{mg} / \mathrm{dL})$ & $144.2 \pm 51.5$ \\
HbA1c (mmol/mol $(\%))$ & $62.4(7.9) \pm 17.2(1.6)$ \\
C peptide (ng/mL) & $0.897 \pm 0.47$ \\
\hline & Per cent of \\
& participants \\
\hline Male & 66.0 \\
Ethnicity & \\
$\quad$ Caucasians & 87.2 \\
African-American & 6.4 \\
Hispanic & 4.3 \\
Asian & 2.1 \\
Comorbidities & \\
Dyslipidemia & 74.5 \\
Hypertension & 73.9 \\
Received insulin therapy & 43.6 \\
\hline BMl, body mass index; HbA1c, glycated hemoglobin; HDL, \\
high-density lipoprotein; LDL, low-density lipoprotein; TG, \\
triglycerides. &
\end{tabular}

\section{Seventeen-week krill oil supplementation}

Of the 47 study participants, 34 completed an additional 17-week krill oil supplementation period before returning for a final assessment visit. On average, participants received the extended period supplementation for 17.4 \pm 1 . 6 weeks with variability due to a participant's availability to return for a final follow-up visit. The results for 17 weeks of krill oil supplementation compared with baseline are provided in table 3 .

The mean EndoPAT RHI for the 17-week krill oil supplementation was statistically significantly higher than the mean baseline for this group $(p=0.04)$, and there was a statistically significant increase in HDL $(p=0.002)$. While a reduction of $\mathrm{C}$ peptide was observed after 4 weeks, this observation was not observed after 17 weeks of krill oil supplementation $(p=0.599)$. Further, when $\mathrm{C}$ peptide was analyzed by grouping insulin therapy and oral glucose-lowering agents separately, there was no statistically significant change observed. Similarly, HOMA2-IR scores were not statistically significantly different from the baseline $(\mathrm{p}=0.874)$.

\section{DISCUSSION}

Our investigation has shown that krill oil supplementation in patients with type 2 diabetes reduces some cardiovascular risk factors by improving endothelial function and increasing serum HDL. $\mathrm{C}$ peptide and insulin 
Table 2 Four weeks krill oil versus olive oil supplementation

\begin{tabular}{|c|c|c|c|}
\hline Measure & $\begin{array}{l}\text { Krill oil } \\
\text { Mean } \pm \text { SD }\end{array}$ & $\begin{array}{l}\text { Olive oil } \\
\text { Mean士SD }\end{array}$ & p Value \\
\hline EndoPAT (RHI) & $2.04 \pm 0.52$ & $1.83 \pm 0.56$ & $0.025^{\star}$ \\
\hline $\mathrm{HbA1c}(\%)$ & $7.6 \pm 1.5$ & $7.7 \pm 1.6$ & 0.300 \\
\hline TC (mg/dL) & $152.2 \pm 30.9$ & $147.5 \pm 30.6$ & 0.078 \\
\hline $\mathrm{HDL}(\mathrm{mg} / \mathrm{dL})$ & $47.34 \pm 13.54$ & $46.35 \pm 15.53$ & 0.528 \\
\hline Triglycerides (mg/dL) & $145.80 \pm 97.62$ & $139.56 \pm 88.16$ & 0.567 \\
\hline $\mathrm{LDL}(\mathrm{mg} / \mathrm{dL})$ & $76.36 \pm 18.36$ & $72.27 \pm 23.48$ & 0.194 \\
\hline Non-HDL (mg/dL) & $102.40 \pm 33.35$ & $102.56 \pm 32.44$ & 0.948 \\
\hline $\mathrm{TC} / \mathrm{HDL}$ & $3.41 \pm 1.07$ & $3.43 \pm 1.15$ & 0.817 \\
\hline Glucose (mg/dL) & $143.41 \pm 49.07$ & $145.87 \pm 57.37$ & 0.723 \\
\hline C peptide (ng/mL) & $0.822 \pm 0.39$ & $0.933 \pm 0.47$ & $0.029^{*}$ \\
\hline With insulin therapy & $0.673 \pm 0.36$ & $0.782 \pm 0.47$ & 0.136 \\
\hline Without insulin therapy & $0.951 \pm 0.37$ & $1.095 \pm 0.43$ & $0.034^{*}$ \\
\hline HOMA-IR & $2.30 \pm 0.91$ & $2.64 \pm 1.23$ & $0.019^{\star}$ \\
\hline Total antioxidant capacity (nM) & $97.55 \pm 11.81$ & $98.26 \pm 10.16$ & 0.431 \\
\hline
\end{tabular}

resistance were also reduced after 4 weeks of supplementation, but was not observed after 17 weeks of supplementation. However, on further analysis, a reduction of $\mathrm{C}$ peptide in patients receiving insulin therapy was not observed, but was in patients receiving oral glucoselowering agents after 4 weeks of supplementation. Because CVD is the most common cause of death in people with type 2 diabetes, both providers and patients seek solutions to minimize cardiovascular risk factors and increase lifespan in this high-risk population. ${ }^{15}$ The reduction of traditionally measured risk factors, such as high cholesterol and hypertension, or those less often measured, such as endothelial dysfunction, can typically be achieved by pharmacological or lifestyle interventions. Endothelial function has been shown to be impaired in patients with type 2 diabetes and is further exacerbated when these patients also have hypertension. ${ }^{16}$ Our sample was at high risk for endothelial dysfunction and CVD, as approximately three out of four participants had both type 2 diabetes and hypertension. ${ }^{16-19}$

Based on the cross-over results, krill oil supplementation may improve endothelial function better than olive oil. The mechanism for this improvement could be attributed to the bioavailability and structure of $\omega-3$ fatty acids in krill oil and its ability to reduce inflammation or other physiological properties, but would require further studies to characterize the mechanism. ${ }^{20}$

Insulin resistance may contribute to the development of endothelial dysfunction and is common among participants with type 2 diabetes. $^{21}$ In addition, HOMA

Table 3 Seventeen-week supplementation with krill oil relative to baseline

\begin{tabular}{lccc}
\hline Measure & $\begin{array}{c}\text { Baseline } \\
\text { Mean } \pm \text { SD }\end{array}$ & $\begin{array}{c}\text { Krill oil } \\
\text { Mean } \pm \text { SD }\end{array}$ & p Value \\
\hline EndoPAT (RHI) & $1.90 \pm 0.54$ & $2.16 \pm 0.73$ & $0.041^{*}$ \\
HbA1c (\%) & $7.5 \pm 1.1$ & $7.4 \pm 1.2$ & 0.388 \\
TC (mg/dL) & $146.38 \pm 29.04$ & $152.56 \pm 27.41$ & 0.242 \\
HDL (mg/dL) & $43.48 \pm 14.10$ & $49.09 \pm 15.63$ & $0.002^{*}$ \\
Triglycerides (mg/dL) & $148.18 \pm 96.35$ & $144.82 \pm 73.79$ & 0.815 \\
LDL (mg/dL) & $76.64 \pm 27.70$ & $75.04 \pm 23.82$ & 0.754 \\
Non-HDL (mg/dL) & $103.97 \pm 30.39$ & $104 \pm 29.01$ & 0.995 \\
TC/HDL & $3.57 \pm 1.15$ & $3.31 \pm 1.11$ & 0.166 \\
Glucose (mg/dL) & $141.60 \pm 42.89$ & $139.58 \pm 43.44$ & 0.782 \\
C peptide (ng/mL) & $0.909 \pm 0.49$ & $0.940 \pm 0.49$ & 0.599 \\
HOMA-IR & $2.40 \pm 1.37$ & $2.38 \pm 1.34$ \\
Total antioxidant capacity $(\mathrm{nM})$ & $101.53 \pm 16.15$ & $98.35 \pm 10.87$ \\
\hline
\end{tabular}

*Significant; $p<0.05$.

HbA1c, glycated hemoglobin; HDL, high-density lipoprotein; HOMA-IR, Homeostatic Model Assessment of Insulin Resistance; LDL, low-density lipoprotein; RHI, Reactive Hyperemia Index; TC, total cholesterol. 
estimated insulin resistance, when measured as cohorts and not individually, may be suggestive of CVD risk in patients with type 2 diabetes. ${ }^{21}{ }^{22}$ A significant reduction of HOMA2-IR, or insulin resistance, in participants receiving krill oil during the initial cross-over supplementation period was observed. While HOMA2-IR cannot be used to make generalized statements about insulin resistance on an individual basis, it is useful when evaluating the effect of a supplement or treatment in a population. ${ }^{11}{ }^{23}$ Insulin activates intracellular signaling pathways that are critical to maintaining a healthy endothelium. Insulin resistance may contribute to deficiencies in the signaling pathway and result in impaired maintenance of the endothelium, such as oxidative stress, inflammatory, and thrombotic effects. ${ }^{16}$ In brief, krill oil's observed reduction of insulin resistance or HOMA2-IR may provide a short-term mechanism to maintain healthy endothelium, but may not be responsible for the overall improvement of endothelial function observed in this trial and may only apply to patients who are not receiving insulin therapy. In addition, future studies should consider other methods to evaluate insulin resistance to confirm this finding due to HOMA2-IR's existing limitations around the interpretation of individual results.

A significant improvement in serum HDL was observed following 17 weeks of krill oil supplementation in participants with type 2 diabetes. Though this effect was not observed during the 4-week cross-over period of the trial, the sustained use of krill oil may increase the serum HDL in type 2 diabetes participants. An increase of HDL in patients with type 2 diabetes may reduce the risk for CVD, especially when patients are diagnosed with other comorbidities causing an increased risk of CVD, like hypertension or dyslipidemia $(73.9 \%$ and $74.5 \%$ of participants enrolled, respectively). ${ }^{8}$ The observed improvement of EndoPAT and HDL after 17 weeks suggests that future studies should consider supplementation periods longer than 4 weeks.

This study included a free-living design allowing the participants to control their diets (including other fatty fish consumption) and activity to simulate krill oil consumption and its potential effect in patients in the uncontrollable world. ${ }^{4}$ To be able to compare participants despite their differences in lifestyles, participants acted as their own controls for comparison and none reported any changes in their diet or level of activity throughout the duration of the study. The krill oil still displayed significant improvement in endothelial function and blood HDL levels, despite the variability between subject lifestyles and clinical histories. Our results suggest that krill oil supplementation may provide similar benefits to patients outside of this clinical study regardless of lifestyle differences. These results are a conservative estimate and thus, if the diets of participants were restricted and they were placed on an exercise regimen, the effect of krill oil supplementation could be even greater.
Further, olive oil contains a plant-derived $\omega-3$ fatty acid called $\alpha$-linolenic acid (ALA) which is known to have strong health benefits. ${ }^{24}$ The dose of ALA in olive oil consumed by participants in this study was $8 \mathrm{mg}$ /day and is below the minimum dosage of $\omega-3$ fatty acids recommended by the AHA to reduce the risk of developing CVD. While using olive oil in the cross-over design may appear as a limitation, the margin of improvement when comparing the results of olive oil and krill oil actually narrows. This suggests that if something more benign were used instead, like water, the improvement of cardiovascular risk factors may have been even greater after supplementation with krill oil.

This study also sought to evaluate krill oil's effect on glucose control. HbA1c represents a patient's glucose control over the 2-3 months prior to testing. Thus, while the 4 weeks in the cross-over design was not long enough to evaluate krill oil's effect on glucose control, 17 weeks is sufficient time and there was no significant improvement.

Additional longitudinal studies must be performed to confirm our finding that krill oil reduces some cardiovascular risks in patients with type 2 diabetes. Unlike other similar studies, this study did not measure participants' blood pressures at each time point, despite the high proportion of patients with hypertension in this study. ${ }^{34}$ Future studies should include methods to better evaluate insulin resistance and include blood pressure measurements. Krill oil supplementation should also be compared with the effect of prescription $\omega$-3-acid ethyl esters products, like Lovaza, as krill oil supplementation may be a less expensive option for some patients. It is also important to note that the US FDA does not require approval for supplements, so thorough investigation of supplement effects on patients is important. ${ }^{25}$

Acknowledgements The authors of the trial wish to acknowledge Joseph Belsky, MD for his help with trial implementation, Tannaz Sedghi, Kyaw Sint, MPH and Mahshid Mohammadi, MD for their statistical expertise, and Kara Crowley, MS for her support and assistance.

Contributors JML, ADB, JRP, and RA were responsible for conception and trial design. JML, LCD, ADB, and MEC were responsible for recruitment and trial visits. JML was responsible for the coordination of the trial. JML, LCD, and $A D B$ performed sample analysis, and JML, ADB, and JRP were responsible for statistical analysis. JML, JRP, and RA undertook data interpretation. JML and JRP were responsible for writing the manuscript. Finally, all authors critically reviewed the manuscript.

Funding This work was supported by Prograde, Inc (Lutz, Florida, USA). Prograde, Inc provided the krill oil and olive oil supplements and funding for the study.

Competing interests None declared.

Ethics approval Biomedical Research Alliance of New York (BRANY).

Provenance and peer review Not commissioned; externally peer reviewed.

Data sharing statement Data may be shared on request.

Open Access This is an Open Access article distributed in accordance with the Creative Commons Attribution Non Commercial (CC BY-NC 4.0) license, which permits others to distribute, remix, adapt, build upon this work noncommercially, and license their derivative works on different terms, provided the original work is properly cited and the use is non-commercial. See: http:// creativecommons.org/licenses/by-nc/4.0/ 


\section{REFERENCES}

1. Kris-Etherton PM, Harris WS, Appel LJ. Fish consumption, fish oil, omega-3 fatty acids, and cardiovascular disease. Circulation 2002;106:2747-57.

2. Deutsch L. Evaluation of the effect of Neptune Krill Oil on chronic inflammation and arthritic symptoms. J Am Coll Nutr 2007;26:39-48.

3. Lorber D. Importance of cardiovascular disease risk management in patients with type 2 diabetes mellitus. Diabetes Metab Syndr Obes 2014:7:169-83.

4. Schuchardt JP, Schneider I, Meyer $\mathrm{H}$, et al. Incorporation of EPA and DHA into plasma phospholipids in response to different omega-3 fatty acid formulations- a comparative bioavailability study of fish oil vs. krill oil. Lipids Health Dis 2011;10:145.

5. Laidlaw M, Cockerline CA, Rowe WJ. A randomized clinical trial to determine the efficacy of manufacturers' recommended doses of omega-3 fatty acids from different sources in facilitating cardiovascular disease risk reduction. Lipids Health Dis 2014;13:99.

6. Ramprasath VR, Eyal I, Zchut S, et al. Enhanced increase of omega-3 index in healthy individuals with response to 4-week n-3 fatty acid supplementation from krill oil versus fish oil. Lipids Health Dis 2013;12:1784.

7. Backes JM, Howard PA. Krill oil for cardiovascular risk protection: is it for real? Hosp Pharm 2014;49:907-12.

8. Fasset RG, Coombes JS. Astaxanthin in cardiovascular health and disease. Molecules 2012;17:2030-48.

9. Ji L, Hu D, Pan C, et al. Primacy of the 3B approach to control risk factors for cardiovascular disease in type 2 diabetes patients. $A m \mathrm{~J}$ Med 2013;126:925.e11-22.

10. Wellek S, Blettner M. On the proper use of the cross-over design in clinical trials: part 18 of a series on evaluation of scientific publications. Dtsch Arztebl Int 2012;109:276-81.

11. Hamburg $\mathrm{N}$, Keyes $\mathrm{M}$, Larson $\mathrm{M}$, et al. Cross-sectional relations of digital vascular function to cardiovascular risk factors in the Framingham Heart Trial. Circulation 2008;117:2467-74.

12. Jones AG, Hattersley AT. The clinical utility of C-peptide measurement in the care of patients with diabetes. Diabet Med 2013;30:803-17.

13. Polonsky KS, Licinio-Paixao J, Given BD, et al. Use of biosynthetic human $\mathrm{C}$-peptide in the measurement of insulin secretion rates in normal volunteers and type I diabetic patients. J Clin Invest 1986;77:98-105.

14. Research Randomizer. http://www.randomizer.org (accessed 11 Jun 2012).

15. Wallace TM, Levy JC, Matthews DR. Use and abuse of HOMA modeling. Diabetes Care 2004;27:1487-95.

16. Zhang P. Cardiovascular disease in diabetes. Eur Rev Med Pharcol Sci 2014;18:2205-14.

17. Roberts AC, Porter KE. Cellular and molecular mechanisms of endothelial dysfunction in diabetes. Diabetes Vasc Dis Res 2013;10:472-82.

18. Moerland M, Kales AJ, Schrier L, et al. Evaluation of the EndoPAT as a tool to assess endothelial function. Int $J$ Vasc Med 2012;2012:904141.

19. Wilk G, Osmenda G, Matusik $P$, et al. Endothelial function assessment in atherosclerosis. Pol Arch Med Wewn 2013;123:443-52.

20. Bruno RM, Penno G, Daniele G, et al. Type 2 diabetes mellitus worsens arterial stiffness in hypertensive patients through endothelial dysfunction. Diabetologia 2012;55:1847-55.

21. Pearson TA, Blair SN, Daniels SR, et al. AHA guidelines for primary prevention of cardiovascular disease and stroke: 2002 update: consensus panel guide to comprehensive risk reduction for adult patients without coronary or other atherosclerotic vascular diseases. American Heart Association Science Advisory and Coordinating Committee. Circulation 2002;106:388-91.

22. Lombardo MF, lacopino $\mathrm{P}$, Cuzzola M, et al. Type 2 diabetes mellitus impairs the maturation of endothelial progenitor cells and increases the number of circulating endothelial cells in peripheral blood. Cytometry Part A 2012;81A:856-64.

23. Bonora $\mathrm{E}$, Formentini $\mathrm{G}$, Calcaterra $\mathrm{F}$, et al. HOMA-estimated insulin resistance is an independent predictor of cardiovascular disease in type 2 diabetic subjects. Diabetes Care 2002;25:1135-41.

24. Fleming JA, Kris-Etherton PM. The evidence for $\alpha$-linolenic acid and cardiovascular disease benefits: comparisons with eicosapentaenoic acid and docosahexaenoic acid. Adv Nutr 2014;5:863S-76S

25. Dietary Supplements. 5 May 2014. http://www.fda.gov/Food/ DietarySupplements/default.html 